\title{
ESACCHARIFICATION OF SOME LIGNOCELLULOSIC \\ MATERIALS BY TRICHODERMA AND ASPERGILLUS SPECIES AND CELLULASES ACTIVITY
}

Mohamoud, Sara, M. ${ }^{(1)}$; Abou-Taleb, Khadiga, A. ${ }^{(2)}$; Shaarawy, H. H. ${ }^{(3)}$; Ramadan, E. M. ${ }^{(2)}$ and ElKassas, H. I. ${ }^{(1)}$

1) Department of Agricultural Sciences, Institute of Environmental Studies and Research, Ain shams University 2) Department of Agricultural Microbiology, Faculty of Agriculture, Ain shams University 3) Chemical Engineering Department, Research Division, NRC.

\begin{abstract}
Biodegradation of Lignocellulosic wastes (cotton stalks and corn cobs) was carried out by single and mixed cultures of Trichoderma viride $\mathrm{T} 3$ and three strains of Aspergillus niger with code A2, A4 and An2. Results indicated that biodegradation of cotton stalks and corn cobs by mixed culture gave higher activities for cellulase production and saccarification than single cultures. Mixed culture of Trichoderma viride T3 and Aspergillus niger A4 gave the highest activity of cellulase being $1.02 \& 0.94 \mathrm{U} / \mathrm{ml}$ with $49.7 \&$ $45.8 \%$ of saccarification on media containing alkaline corn cobs and cotton stalks, respectively. It was observed that the ability of the mixed for degrading corn cobs was more efficient than degrading cotton stalks which increased about 1.08 fold of cellulase activity and saccharification. The biological and enzyme parameters of co-culture on alkaline corn cobs were calculated, which recorded $56.73 \%$ of yield factor, $85.59 \%$ of sugar utilization efficiency, $16.60 \%$ of effective yield, $32.55 \%$ enzyme yield, $37.60 \%$ conversion coefficient with productivity of $0.15 \mathrm{U} / \mathrm{ml} / \mathrm{d}$.
\end{abstract}

Keywords: Cellulase activity, Lignocellulosic materials, saccharification, Aspergillus niger, Trichoderma viride. 
J. Environ. Sci.

Institute of Environmental Studies and Research - Ain Shams University

\section{INTRODUCTION}

In Egypt, crop residues are byproducts of common crops such as cotton, wheat, maize and rice, with total amount of about 16 million tons of dry matter per year. Cotton crop area accounts for about $5 \%$ of the cultivated area in Egypt (El Saeidy, 2004).

Cotton stalks produced annually as agricultural residues reached 1.9 million tons (Mona, et al., 2001). These post-harvest byproduct cause many severe problems, fires causing significant environmental and health disorders (Fouad et al., 2010).

As a renewable raw material, corncobs is considered a potential feedstock for the production of biogas, biodiesel and bioethanol to fulfil the increasing demand for biofuels (Ioannidou et al., 2009). The hydrolysates of corncobs are therefore perfectly suited for biodiesel production using yeasts. These species of oil-producing yeasts accumulate up to $50 \%$ of fat in their dry mass (Kitcha and Cheirsilp, 2011).

Corncobs are a lignocellulosic material composed of cellulose, hemicellulose and lignin. These polymeric fibers consist of monomeric molecules. Cellulose is built of C6 sugars; hemicellulose mainly of the C5 sugars (xylose and arabinose). Lignin consists of phenolic macromolecules (Pointner et al., 2014).

Pretreatment aims to decrease crystallinity of cellulose, increase biomass surface area, remove hemicellulose, and break lignin seal. Pretreatment makes cellulose more accessible to enzymes so that conversion of 
carbohydrate polymers into fermentable sugars can be achieved more rapidly and with more yields (McMillan et al., 1994).

The major methods include pretreatment by milling (Delgenés et al., 2002), acid hydrolysis (Taherzadeh and Karimi, 2007), steam explosion (Mukhopadhyay and Fangueiro, 2009), hot water (Liu and Wyman, 2005) and alkaline hydrolysis (Goswami et al., 2009 and Binod et al., 2010). Usually grinding and milling are the initial steps of pretreatment of any biomass which reduces the particle size, though the combination of grinding with other pretreatment method. Superfine grinding of steam exploded biomass has been proved better than ground residue when hydrolyzed though energy required for the process also has to be considered while going for commercial applications (Zhu et al., 2006).

There is a large number of fungi play a vital role to degrade these wastes contain cellulose into sugar such as Trichoderma aureoviride, T.ressi, T.koningii BTS120 and Aspergillus sp. (Bahaa et al., 2011; Fang and Xia, 2013 and Rana et al., 2014).

The aim of the work was to investigate the saccharification of lignocellulosic materials (cotton stalks and corn cobs) into sugar by fungal cultures. The biological parameters of the tested fungi were also elucidated.

\section{MATERIAL AND METHODS}

Fungi used: Three isolates of Aspergillus sp. and one isolate of Trichoderma sp. used in this study, as cellulytic fungi, were obtained from Microbiology Department, Fac. of Agric., Ain Shams University, Cairo, Egypt. 


\section{Media used:}

Medium (1), Potato dextrose agar (PDA), was used for maintenance and preservation of the tested fungi, described by (Difco Manual, 1984). The same medium was used without agar as broth medium.

Medium (2), Basal medium (Mandels et al., 1969). It was used to study cellulase activity and saccharification determination. Its composition was as follows (g/L): Urea, 0.3; $\left(\mathrm{NH}_{4}\right)_{2} \mathrm{SO}_{4}, 1.4 ; \mathrm{KH}_{2} \mathrm{PO}_{4}, 2 ; \mathrm{CaCl}_{2}, 0.3 ; \mathrm{MgSO}_{4}, 0.3$; Yeast extract, 0.25; Peptone, 0.75; Trace elements (mg/L): $\mathrm{FeSO}_{4} .7 \mathrm{H}_{2} \mathrm{O}, 5$; $\mathrm{COCl}_{2}, 20 ; \mathrm{MnSO}_{4}, 1.6$ and $\mathrm{ZnSO}_{4}, 1.4$ then adjusted $\mathrm{pH}$ to 7.0 .

These media were autoclaved at $121^{\circ} \mathrm{C}$ for $15 \mathrm{~min}$.

\section{Buffers and solutions}

- Citrate buffer (0.05M) consists of : Solution (a) 0.05 M citric acid (10.51 $\mathrm{g} / \mathrm{L}$ ) and solution (b) $0.05 \mathrm{M}$ trisodium citrate $(14.71 \mathrm{~g} / \mathrm{L})$, adjusted $\mathrm{pH}$ to 4.8 by adding $667 \mathrm{ml}$ solution (a) to 1 liter of solution (b) (Mandels et al., 1969).

- Carboxymethlycellulase (CMC) solution 1\% (Mandels et al., 1969). Its composition was as follows: CMC $10 \mathrm{~g} / \mathrm{L}$ and adjusted $\mathrm{pH}$ to 4.8 .

Lignocellulosic agricultural wastes: Agricultural wastes (cotton stalks and corn cobs) were collected from Kafr El-Dawar, El-Bahera Governorate.

Cotton stalks and corn cobs were used as samples of agriculture wastes, which harvested from field, and solid biomass was washed with tap water until clean, then dried at $80^{\circ} \mathrm{C}$ overnight (Yonghao et al., 2016). The dried biomass was milled, as mechanical pretreatment (MT1), then using different pretreatments, being thermal pretreatment (TT2) by heating at $121^{\circ} \mathrm{C} / 1 \mathrm{~h}$ and 
physiochemical pretreatment by acid (AT3) was carried out using $\mathrm{H}_{2} \mathrm{SO}_{4} 10 \%$ /1h (Noriko et al., 2005) or alkaline (KT4) was done with $\mathrm{NaOH}$ at concentrations of $10 \%$ for $1 \mathrm{~h}$ (Singh et al., 2011).

The pretreated biomass were filtered with two layers of muslin cloth, and washed with distilled water. Then, biomass was dried at $50 \pm 2{ }^{\circ} \mathrm{C}$ and subsequently used for enzymatic hydrolysis experiments.

Maintenance of cultures: Stocks culture slants were grown on PDA medium at $30^{\circ} \mathrm{C}$ for $3-5$ days and maintained at $5^{\circ} \mathrm{C}$.

Standard inoculants: The tested fungal isolates grown on PDA slants for 3 days at $30^{\circ} \mathrm{C}$ and were used to prepare the spore suspensions by adding $10 \mathrm{ml}$ of sterile saline solution $(0.95 \% \mathrm{NaCl})$ water to each fungal agar slant and gently scraping with sterile inoculation loop. The obtained spore suspensions $\left(1 \times 10^{10} / \mathrm{ml}\right)$ were used as fungal standard inoculants for flasks experiments.

Submerged fermentation process for saccharification (cellulase production): It was carried out in $250 \mathrm{ml}$ plugged Erlenmeyer flasks containing $100 \mathrm{ml}$ of basal medium supplemented with $1 \%(\mathrm{w} / \mathrm{v})$ pretreated agricultural waste samples and then inoculated with $5 \%(\mathrm{v} / \mathrm{v})$ of standard inoculants of the tested fungal isolates. The inoculated flasks were incubated at $30^{\circ} \mathrm{C}$ on a rotary shaker at $100 \mathrm{rpm} / \min$ for 7 days.

The fermented medium was filtrated through whatman No.1 filter paper to separate mycelial mat to determine the cell dry weight. The culture filtrate was used to determine enzyme activity, extracellular consumed sugar. Some biological parameters were calculated (Diener et al., 2004). 
J. Environ. Sci.

Institute of Environmental Studies and Research - Ain Shams University

Identification of pioneer tested fungal isolates: The most efficient fungal isolates for saccharification were identified based on the morphological appearance under light microscope (shape and conidia) and cultural characteristics according to Barnett and Hunter (1998).

\section{Analytical procedures}

Fungal count: The number of fungal spores was counted in the filtrate using haemocytometer slide (Kolmer et al., 1959). Fungal dry weight was determined by separate the mycelium from broth culture using filter paper (Whatman No.1) and drying at $80^{\circ} \mathrm{C}$ to constant weight.

Dry cell mass determination (Srilekha et al., 2011):For dry cell mass determination, $10 \mathrm{ml}$ of culture samples were filtered, washed and dried to a constant mass at $104^{\circ} \mathrm{C}$.

pH determination: $\mathrm{pH}$ of culture was measured using $\mathrm{pH}$-meter model (Microprocessor 211) equipped with glass electrode.

Reducing sugar determination: Glucose was determined using glucose oxidase peroxidase kits (GOD-POD. Liquid) from EL NASER PHARMACEUTICAL CHEMICALS CO. (Egypt) using spectrophotometer (JENWAY 6300) and measured at $546 \mathrm{~nm}$ (Kaplan et al., 2001).

Enzyme assay: Carboxymethyl-cellulase (CMCase) activity was assayed using a method suggested by Mandels et al. (1962). The activity was estimated using $1 \%$ solution of carboxymethlycellulase (CMC) in $0.05 \mathrm{M}$ citrate buffer $(\mathrm{pH} 4.8)$ as a substrate. The reaction mixture contained $1 \mathrm{ml}$ citrate buffer, $0.5 \mathrm{ml}$ of substrate solution and $0.5 \mathrm{ml}$ of suitably diluted enzyme solution. The reaction was carried out at $50^{\circ} \mathrm{C}$ for $30 \mathrm{~min}$. The 
amount of reducing sugar released in the hydrolysis was measured. One unit of CMCase activity was expressed as $1 \mu \mathrm{mol}$ of glucose liberated per $\mathrm{ml}$ enzyme per minute.

\section{Parameters calculation:}

Yield factor $(Y)$ (Herbert et al., 1971) $=(($ Growth $($ dry weight $) /$ Consumed sugar)) $\times 100$

Enzyme Yield (EY) (Ramadan et al., 1985) $=(($ Enzyme activity $(\mathrm{U} / \mathrm{ml}) /$ Original sugar $\left.\left(\mathrm{gl}^{-1}\right)\right) \times 100$

Effective Yield $(\mathbf{Y E})=(($ Fungal biomass $) /($ Initial substrate concentration $))$ $\times 100$

Substrate Utilization Efficiency (SUE) $=(($ Effective yield $($ YE) $/$ Yield factor $(\mathrm{Y})) \times 100$

Sugar utilization efficiency $($ Ramadan et al., 1985) $=($ Consumed sugar $/$ Initial sugar)

Productivity (P) (Lee et al., 1996) $=\left(\left(\right.\right.$ Enzyme activity $\left(\mathrm{Uml}^{-1}\right) /$ Fermentation time (h))

Conversion coefficient $(\mathbf{C C})=\left(\left(\right.\right.$ Enzyme activity $\left(\mathrm{Uml}^{-1}\right) /$ Utilized sugar $\left.\left(\mathrm{gl}^{-1)}\right)\right) \times 100$

Saccharification Conversion (SC) (Velayudhan et al., 2104) $=(($ Enzyme activity $\mathrm{mg} / \mathrm{ml}) \times 0.9) /($ Initial Substrate concentration $)) \times 100$

Statistical experimental analysis: The collected data were statistically analyzed using IBM® SPSS ${ }^{\circledR}$ Statistics software (2011). The correlation coefficient was analyzed using Microsoft Office Excel 2013. 
J. Environ. Sci.

Institute of Environmental Studies and Research - Ain Shams University

\section{RESULTS AND DISCUSSION}

\section{Collection and identification of fungal isolates:}

In the present study, 4 fungal isolates were collected from Microbiology Dept., Fac. of Agric., Ain shams Univ. They were used as cellulase producers. These isolates belong to Trichoderma sp. with code T3 and Aspergillus sp. with codes A2, A4 and An2.

These fungal isolates were identified depending on their cultural and morphological characteristic. Trichoderma sp. T3 isolate was classified as Trichoderma viride, which giving colonies on PDA agar with broadly spreading, hyaline with fruiting areas appeared as tufts, white at first and becoming in deep green shades with colorless reverse. Conidiophores arised as branches of mycelium, dichotomously branched, occasionally forming whorls (Fig. 1d).

Whereas, all Aspergillus sp. isolates with codes A2, A4 and An2 were identified as Aspergillus niger, which giving colonies on PDA agar medium rapidly growing with abundant submerged mycelium. Reverse usually without color. Conidiophores mostly arise directly from substratum, conidial heads fuscous, blackish brown, small, most columnar masses of a few conidial chains (Fig. 1a-c).

Among the filamentous fungi of environmental importance are Trichoderma sp. and Aspergillus sp., their hydrolytic efficiency is as a result of secretion of extracellular enzymes such as cellulases, hemicellulases and ligninases. Of these, cellulase is the most important and a complex enzyme 
that acts synergistically though often described as contrasting faces of a single enzymatic capability (Milala et al., 2014).

Pretreatment makes cellulose more accessible to enzymes so that conversion of carbohydrate polymers into fermentable sugars can be achieved more rapidly and with more yields (McMillan et al., 1994).

Therefore, an experiment was carried out to investigate the effect of four different pretreatments of agricultural wastes (cotton stalks and corn cobs) on saccharification and cellulase production by single and mixed fungi (Tables 1-2). It was found that lignocellulosic wastes were more biodegradable by mixed fungal cultures (Trichoderma viride T3 \& Aspergillus niger A2; Trichoderma viride T3 \& Aspergillus niger A4 or Trichoderma viride T3 \& Aspergillus niger An2) than single culture (Trichoderma viride T3, Aspergillus niger A2, Aspergillus niger A4 or Aspergillus niger An2), it could be due to produce a large and more efficient enzymes necessary to breakdown hemicelluloses and cellulose (Nigam et al., 2009).

Results demonstrated that the co-culture of Trichoderma viride T3 \& Aspergillus niger A2, Trichoderma viride T3 \& Aspergillus niger A4 or Trichoderma viride $\mathrm{T} 3$ \& Aspergillus niger An2 achieved the highest degradation significant effect of pretreatments cotton stalks and corn cobs, which gave the maximum yield of biomass ranged from $0.90-1.39 \mathrm{~g} / \mathrm{L}$ and $1.00-1.66 \mathrm{~g} / \mathrm{L}$, sugar consumption between $2.38-2.64 \mathrm{~g} / \mathrm{L}$ and $2.72-2.97$ $\mathrm{g} / \mathrm{L}$ and cellulase activity ranged from $0.67-0.94 \mathrm{U} / \mathrm{ml}$ and $0.70-1.02 \mathrm{U} / \mathrm{ml}$ with $\%$ of saccharification ranged from $\%, 32.5-45.8 \%$ and $34.1-49.7 \%$, respectivly 
J. Environ. Sci.

Institute of Environmental Studies and Research - Ain Shams University
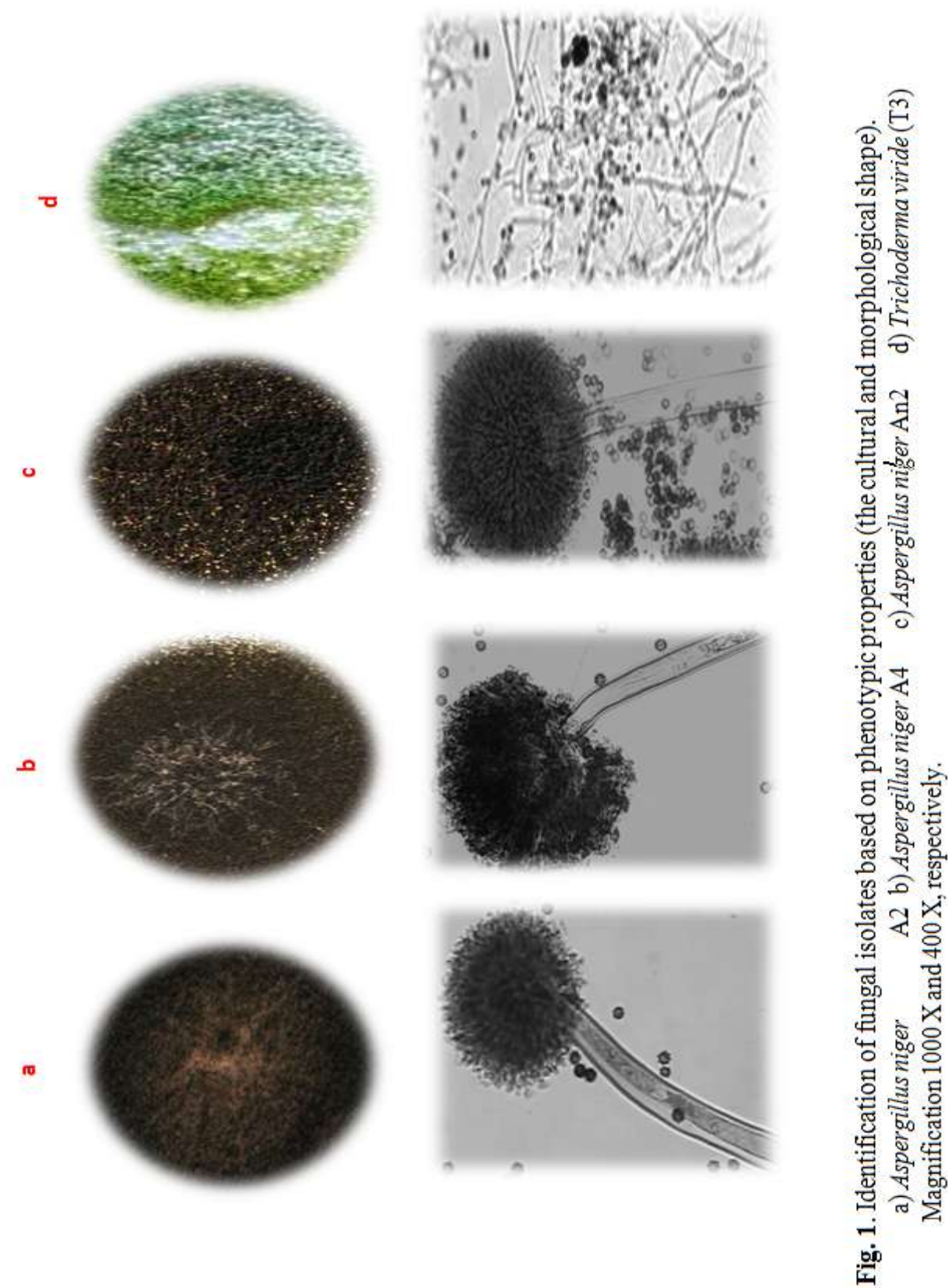
Furthermore, results indicated that the highest biodegradation (saccharification, biomass and enzyme activity) were achieved by the tested fungi when propagated on wastes after pretreatment with physicochemical by 10 $\% \mathrm{NaOH}$ (KT4 treatment) followed by $10 \% \mathrm{H}_{2} \mathrm{SO}_{4}$ (AT3 treatment), then physical treatment with heat at $121^{\circ} \mathrm{C}$ (TT2 treatment) and milling (MT1 treatment). So, it was observed that physicochemical pretreatment of cotton stalks and corn cobs with $10 \% \mathrm{NaOH}$ was the best one for biomass production being ranged from $1.28-1.39 \mathrm{~g} / \mathrm{L}$ and $1.37-1.66 \mathrm{~g} / \mathrm{L}$ with saccharification ranged from $41.7-45.8 \%$ and $41.8-49.7 \%$ and enzyme activity ranged from $0.86-0.94 \mathrm{U} / \mathrm{ml}$ and $0.86-1.02 \mathrm{U} / \mathrm{ml}$, respectively.

The co-culture of $T$. viride $\mathrm{T} 3$ and $A$. niger $\mathrm{A} 4$ gave the maximum saccharification (45.8 and $49.7 \%$ ), biomass (1.39 and $1.66 \mathrm{~g} / \mathrm{L})$ and enzyme activity $(0.94$ and $1.02 \mathrm{U} / \mathrm{ml})$ on media supplemented with alkaline pretreatment of cotton stalks and corn cobs, respectively.

Moreover, it was observed that the saccharification and enzyme activity by mixed fungi $T$. viride $\mathrm{T} 3 \&$ A. niger $\mathrm{A} 4$ on alkaline wastes of cotton stalks and corn cobs were high significant at $p \leq 0.05$ and increased about $\simeq 1.2$ folds as compared to single fungal culture of $T$. viride $\mathrm{T} 3$ or A. niger $\mathrm{A} 4$, respectively.

The biological activity of the tested fungi (single and mixed) were calculated and illustrated by Figs. (2-3). The highest figures of all calculated parameters of yield factor, effective yield, substrate utilization efficiency, sugar utilization efficiency, conversion coefficient, enzyme yield and productivity were recorded by co-culture of $T$. viride $\mathrm{T} 3 \&$ A. niger A4 on all 
pretreatments lignocelluloses waste as compared with another the tested fungi.

The maximum parameters were obtained by $T$. viride $\mathrm{T} 3 \&$ A. niger A4 on alkaline pretreatment (KT4 treatment) of cotton stalks and corn cobs being 55.19 and $56.73 \%$ of yield factor, 13.85 and $16.60 \%$ of effective yield and $84.08,85.59 \%$ of sugar utilization efficiency and $26.4,29.7 \%$ of substrate utilization efficiency, respectively. Moreover, the highest productivity and enzyme yield were 0.14 and $0.15 \mathrm{U} / \mathrm{ml} / \mathrm{d}, 30.06$ and $32.55 \%$ with conversion coefficient being 34.93 and $37.60 \%$ on cotton stalks and corn cobs, respectively.

The main effect of sodium hydroxide pretreatment on lignocellulosic biomass is delignification by breaking the ester bonds cross-linking lignin and xylan, thus increasing the porosity of biomass (Tarkov and Feist, 1969).

Results in Table (3), confirmed that mixed cultures of $T$. viride T3 \& A. niger A4 breakdown alkaline corn cobs with high efficiency than alkaline cotton stalks which gave a high values of biomass (1.66 g/L), enzyme activity $(1.02 \mathrm{U} / \mathrm{ml})$ and \% saccharification $(49.7 \%)$. From statistically analysis, it was observed a high positive correlation coefficient ( $\mathrm{r}$ ) between biomass and each of saccharification and cellulase production by selected mixed culture of T. viride $\mathrm{T} 3 \&$ A. niger A4 on alkaline pretreatment of cotton stalks and corn cobs ranged from $0.92-0.93$ and $0.88-0.89$, respectively. 
Table (1): Physicochemical pretreatments of cotton stalks for biomass, consumed sugar and cellulase production by single and mixed fungi using flasks as a batch culture at $30^{\circ} \mathrm{C}$ for 7 days.

\begin{tabular}{|c|c|c|c|c|c|c|c|c|c|}
\hline \multirow{3}{*}{ 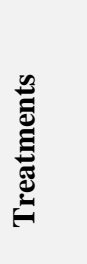 } & & \multicolumn{7}{|c|}{ Tested fungi } & \multirow[b]{3}{*}{ 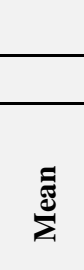 } \\
\hline & & \multicolumn{4}{|c|}{ Single } & \multicolumn{3}{|c|}{ Mixture } & \\
\hline & Parameters & $\mathscr{m}$ & 㫣 & 2 & $\stackrel{\mho}{\gtrless}$ & 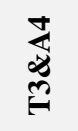 & 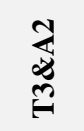 & 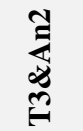 & \\
\hline & C.D.W(g/L) & 0.61 & 0.69 & 0.65 & 0.66 & 1.15 & 1.1 & 1.0 & $0.83^{\mathrm{d}}$ \\
\hline & Saccharification (\%) & 28.42 & 34.1 & 30.4 & 30.1 & 35.7 & 34.0 & 32.5 & $32.2^{\mathrm{c}}$ \\
\hline \multirow[t]{4}{*}{ MT1 } & Consumed sugar $(\mathrm{g} / \mathrm{L})$ & 2.22 & 2.26 & 2.27 & 2.25 & 2.54 & 2.43 & 2.45 & $2.34^{\mathrm{b}}$ \\
\hline & CMC activity(U/ml) & 0.58 & 0.7 & 0.63 & 0.62 & 0.74 & 0.7 & 0.67 & $0.66^{\mathrm{c}}$ \\
\hline & C.D.W $\left(\mathrm{g} / \mathrm{l}^{-1}\right)$ & 0.88 & 0.89 & 0.75 & 0.81 & 1.21 & 1.00 & 0.90 & $0.91^{\mathrm{c}}$ \\
\hline & Saccharification (\%) & 38.43 & 40.78 & 40.24 & 39.4 & 42.7 & 41.5 & 42.0 & $34.7^{\mathrm{b}}$ \\
\hline \multirow[t]{4}{*}{ TT2 } & Consumed sugar(g/L) & 2.48 & 2.49 & 2.5 & 2.51 & 2.64 & 2.53 & 2.53 & $2.36^{\mathrm{b}}$ \\
\hline & CMC activity $(\mathrm{U} / \mathrm{ml})$ & 0.79 & 0.84 & 0.83 & 0.81 & 0.88 & 0.85 & 0.86 & $0.71^{\mathrm{b}}$ \\
\hline & C.D.W $\left(\mathrm{g} / \mathrm{l}^{-1}\right)$ & 0.96 & 0.98 & 0.84 & 0.84 & 1.25 & 1.06 & 1.03 & $0.99^{\mathrm{b}}$ \\
\hline & Saccharification (\%) & 30.73 & 35.0 & 33.4 & 33.9 & 36.9 & 36.6 & 36.7 & $40.3^{\mathrm{a}}$ \\
\hline \multirow[t]{4}{*}{ AT3 } & Consumed sugar(g/L) & 2.42 & 2.31 & 2.24 & 2.11 & 2.61 & 2.46 & 2.38 & $2.38^{\mathrm{b}}$ \\
\hline & CMC activity(U/ml) & 0.63 & 0.72 & 0.69 & 0.7 & 0.76 & 0.75 & 0.76 & $0.83^{\mathrm{a}}$ \\
\hline & C.D.W $\left(\mathrm{g} / \mathrm{l}^{-1}\right)$ & 1.0 & 1.16 & 1.0 & 1.1 & 1.39 & 1.31 & 1.28 & $1.17^{\mathrm{a}}$ \\
\hline & Saccharification (\%) & 36.9 & 39.2 & 38.0 & 37.8 & 45.8 & 42.4 & 41.7 & $40.7^{\mathrm{a}}$ \\
\hline \multirow[t]{2}{*}{ KT4 } & Consumed sugar(g/L) & 2.45 & 2.41 & 2.27 & 2.16 & 2.51 & 2.46 & 2.43 & $2.52^{\mathrm{a}}$ \\
\hline & CMC activity(U/ml) & 0.76 & 0.81 & 0.78 & 0.78 & 0.94 & 0.87 & 0.86 & $0.84^{\mathrm{a}}$ \\
\hline \multirow{4}{*}{ 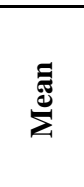 } & C.D.W(g/L) & $0.92^{\mathrm{d}}$ & $0.86^{\mathrm{e}}$ & $0.84^{\mathrm{e}}$ & $0.8^{\mathrm{e}}$ & $1.2^{\mathrm{a}}$ & $1.1^{\mathrm{b}}$ & $1.0^{\mathrm{c}}$ & \\
\hline & Saccharification (\%) & $37.2^{\mathrm{c}}$ & $35.5^{\mathrm{d}}$ & $35.2^{\mathrm{d}}$ & $33^{\mathrm{e}}$ & $40^{\mathrm{a}}$ & $38.6^{\mathrm{b}}$ & $38^{\mathrm{b}}$ & \\
\hline & Con. Sugar $(\mathrm{g} / \mathrm{L})$ & $0.76^{\mathrm{c}}$ & $0.73^{\mathrm{d}}$ & $0.72^{\mathrm{d}}$ & $0.7^{\mathrm{e}}$ & $0.8^{\mathrm{a}}$ & $0.8^{\mathrm{b}}$ & $0.8^{\mathrm{b}}$ & \\
\hline & CMC activity $(\mathrm{U} / \mathrm{ml})$ & $2.4 c^{\mathrm{d}}$ & $2.3^{\mathrm{d}}$ & $2.32^{\mathrm{e}}$ & $2.2^{\mathrm{f}}$ & $2.5^{\mathrm{a}}$ & $2.4^{\mathrm{b}}$ & $2^{\mathrm{b}, \mathrm{c}}$ & \\
\hline
\end{tabular}

*Initial total sugar of pretreated cotton stalks $=3.14(\mathrm{~g} / \mathrm{L}), \mathrm{CMC}=$ carboxy methyl cellulose, C.D.W. = cell dry weight, Con . sugar $=$ consumed sugar, MT1 = mechanical treatment, TT2 $=$ thermal treatment, $\mathrm{AT} 3=$ acid treatment, $\mathrm{KT} 4=$ alkaline treatment, $\mathrm{T} 3=T$. viride, $\mathrm{A} 4=A$. niger $\mathrm{A} 4, \mathrm{~A} 2=A$. niger $\mathrm{A} 2, \mathrm{An} 2=$ A. niger An2. Values are means of 3 replica, Values in the same column followed by same letter do not significantly different from each other, according to Duncan's (1955) at $5 \%$ level. 
J. Environ. Sci.

Institute of Environmental Studies and Research - Ain Shams University

Table (2): Physicochemical pretreatments of corn cobs for biomass, consumed sugar and cellulase production by single and mixed fungi using flasks as a batch culture at $30^{\circ} \mathrm{C}$ for 7 days.

\begin{tabular}{|c|c|c|c|c|c|c|c|c|c|}
\hline \multirow{3}{*}{ } & \multirow[b]{3}{*}{ Parameters } & \multicolumn{7}{|c|}{ Tested fungi } & \multirow[b]{3}{*}{ 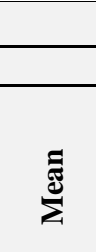 } \\
\hline & & \multicolumn{4}{|c|}{ Single } & \multicolumn{3}{|c|}{ Mixture } & \\
\hline & & $\tilde{\theta}$ & ¿ & 2 & ฏ & $\underset{\mathbb{\infty}}{\mathbb{\infty}}$ & 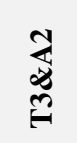 & 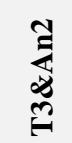 & \\
\hline & C.D.W(g/L) & 0.62 & 0.67 & 0.63 & 0.62 & 1.2 & 1.0 & 1.0 & $0.81^{\mathrm{c}}$ \\
\hline & Saccharification (\%) & 28.48 & 34.15 & 30.5 & 30.1 & 36.2 & 34.6 & 34.1 & $31.23^{\mathrm{c}}$ \\
\hline \multirow[t]{4}{*}{ MT1 } & $\begin{array}{l}\text { Consumed } \\
\operatorname{sugar}(\mathrm{g} / \mathrm{L})\end{array}$ & 2.56 & 2.58 & 2.61 & 2.58 & 2.88 & 2.76 & 2.79 & $2.67^{\mathrm{c}}$ \\
\hline & CMC activity $(\mathrm{U} / \mathrm{ml})$ & 0.59 & 0.74 & 0.68 & 0.65 & 0.74 & 0.73 & 0.70 & $0.66^{\mathrm{d}}$ \\
\hline & C.D.W(g/L) & 0.74 & 0.77 & 0.67 & 0.67 & 1.33 & 1.18 & 1.1 & $0.91^{\mathrm{b}, \mathrm{c}}$ \\
\hline & Saccharification (\%) & 39.33 & 41.3 & 40.8 & 39.2 & 43.6 & 42.4 & 39.9 & $34.64^{b}$ \\
\hline \multirow[t]{4}{*}{ TT2 } & $\begin{array}{l}\text { Consumed } \\
\text { sugar(g/L) }\end{array}$ & 2.83 & 2.85 & 2.87 & 2.86 & 2.97 & 2.92 & 2.94 & $2.73^{\mathrm{b}, \mathrm{c}}$ \\
\hline & CMC activity(U/ml) & 0.81 & 0.85 & 0.84 & 0.81 & 0.9 & 0.87 & 0.87 & $0.73^{\mathrm{c}}$ \\
\hline & C.D.W(g/L) & 0.78 & 0.83 & 0.78 & 0.78 & 1.45 & 1.35 & 1.39 & $1.05^{\mathrm{a}, \mathrm{b}}$ \\
\hline & Saccharification $(\%)$ & 33.3 & $35 . .9$ & 35.1 & 34.3 & 38.3 & 36.9 & 36.0 & $39.1^{\mathrm{a}, \mathrm{b}}$ \\
\hline \multirow[t]{4}{*}{ AT3 } & $\begin{array}{l}\text { Consumed } \\
\text { sugar }(\mathrm{g} / \mathrm{L})\end{array}$ & 2.82 & 2.75 & 2.65 & 2.53 & 2.82 & 2.81 & 2.72 & $2.78^{\mathrm{b}}$ \\
\hline & $\mathrm{CMC}$ activity $(\mathrm{U} / \mathrm{ml})$ & 0.68 & 0.74 & 0.72 & 0.71 & 0.79 & 0.76 & 0.77 & $0.84^{\mathrm{a}, \mathrm{b}}$ \\
\hline & C.D.W(g/L) & 0.93 & 0.94 & 0.89 & 0.88 & 1.66 & 1.4 & 1.37 & $1.15^{\mathrm{a}}$ \\
\hline & Saccharification (\%) & 42.12 & 42.3 & 39.3 & 40.58 & 49.7 & 42.1 & 41.8 & $42.8^{\mathrm{a}}$ \\
\hline \multirow[t]{2}{*}{ KT4 } & $\begin{array}{l}\text { Consumed } \\
\text { sugar }(\mathrm{g} / \mathrm{L})\end{array}$ & 2.85 & 2.8 & 2.77 & 2.59 & 2.93 & 2.81 & 2.79 & $2.89^{\mathrm{a}}$ \\
\hline & CMC activity(U/ml) & 0.87 & 0.87 & 0.81 & 0.83 & 1.02 & 0.86 & 0.88 & $0.88^{\mathrm{a}}$ \\
\hline \multirow{4}{*}{ 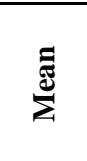 } & C.D.W(g/L) & $0.8^{\mathrm{c}}$ & $0.76^{\mathrm{c}}$ & $0.7^{\mathrm{c}}$ & $0.72^{\mathrm{c}}$ & $1.4^{\mathrm{a}}$ & $1.2^{\mathrm{b}}$ & \multicolumn{2}{|c|}{$1.1^{\mathrm{a}, \mathrm{b}}$} \\
\hline & Saccharification (\%) & $36.4^{b}$ & $36^{b}$ & $35.6^{b}$ & $30.5^{\mathrm{c}}$ & $41^{\mathrm{a}}$ & $39^{\mathrm{a}}$ & \multicolumn{2}{|c|}{$38.4^{\mathrm{a}}$} \\
\hline & Con. Sugar (g/L) & $2.7^{\mathrm{b}, \mathrm{c}}$ & $2.7^{\mathrm{b}, \mathrm{c}}$ & $2.6^{\mathrm{b}, \mathrm{c}}$ & $2.58^{\mathrm{c}}$ & $2.9^{\mathrm{a}}$ & $2.8^{\mathrm{b}}$ & \multicolumn{2}{|c|}{$2.8^{\mathrm{a}, \mathrm{b}}$} \\
\hline & CMC activity $(\mathrm{U} / \mathrm{ml})$ & $0.68^{\mathrm{c}}$ & $0.67^{\mathrm{c}}$ & $0.67^{\mathrm{c}}$ & $0.67^{\mathrm{c}}$ & $0.8^{\mathrm{a}}$ & $0.7^{\mathrm{b}}$ & \multicolumn{2}{|c|}{$0.67^{\mathrm{b}}$} \\
\hline
\end{tabular}

$*$ Initial total sugar of pretreated corn cobs $=3.47(\mathrm{~g} / \mathrm{L}), \mathrm{CMC}=$ carboxy methyl cellulose, C.D.W. = cell dry weight, Con. sugar = consumed sugar, MT1 = mechanical treatment, $\mathrm{TT} 2=$ thermal treatment, $\mathrm{AT} 3=$ acid treatment, $\mathrm{KT} 4=$ alkaline treatment, $\mathrm{T} 3=T$. viride, $\mathrm{A} 4=A$. niger $\mathrm{A} 4, \mathrm{~A} 2=A$. niger $\mathrm{A} 2, \mathrm{An} 2=A$. niger An2. Values are means of 3 replica, Values in the same column followed by same letter do not significantly different from each other, according to Duncan's (1955) at $5 \%$ level 


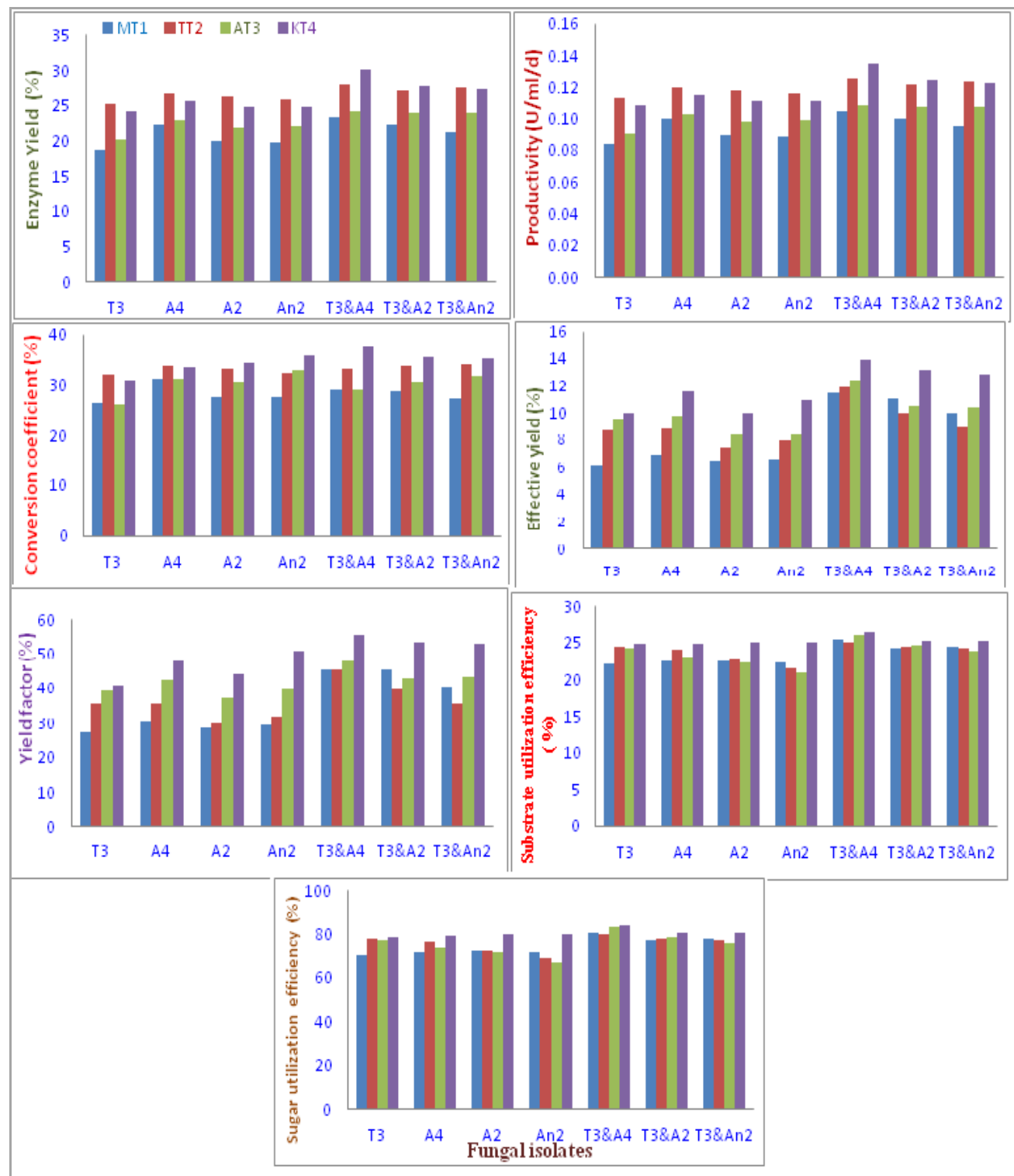

Fig. (2): Biological and enzyme activity parameters of single and mixed tested fungi on media containing of cotton stalks after mechanical (MT1), thermal (TT2), acid (AT3) and alkaline (KT4) treatments using flasks as batch culture for 7 days at $30^{\circ} \mathrm{C}$. 
J. Environ. Sci.

Institute of Environmental Studies and Research - Ain Shams University

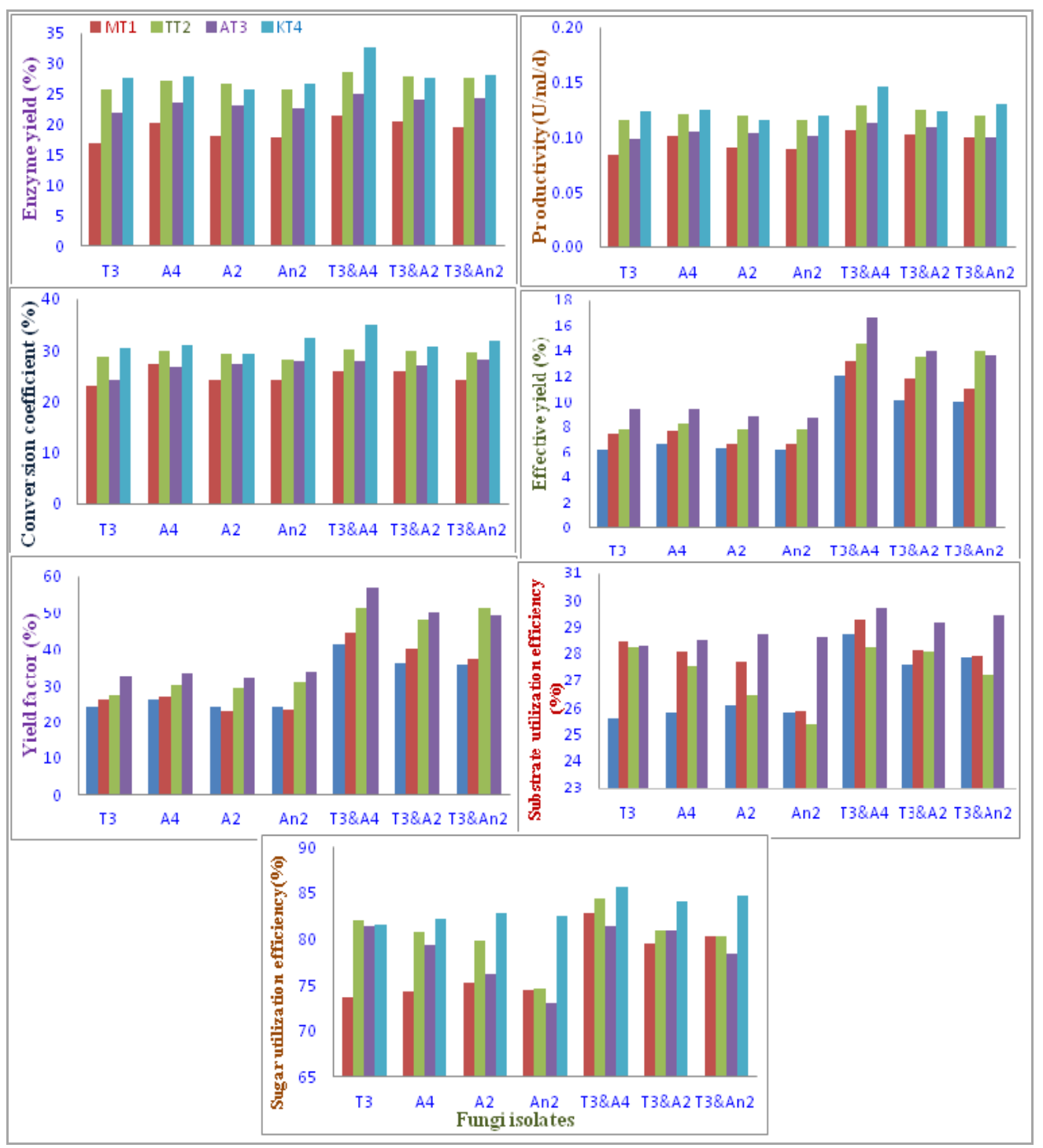

Fig. (3): Biological and enzyme activity parameters of single and mixed tested fungi on media containing of corn cobs after mechanical (MT1), thermal (TT2), acid (AT3) and alkaline (KT4) treatments using flasks as batch culture for 7 days at $30^{\circ} \mathrm{C}$ 
Table (3): Comparative data for biomass, saccharification and cellulase production by $T$. viride $\mathrm{T} 3 \&$ A. niger $\mathrm{A} 4$ as influence by alkaline pretreatment of lignocellulosic wastes

\begin{tabular}{|c|c|c|c|c|c|}
\hline \multirow[b]{2}{*}{ Parameters } & \multicolumn{4}{|c|}{ Alkaline hydrolyzed agro-industrial wastes } & \multirow[b]{2}{*}{ rBs } \\
\hline & $\begin{array}{c}\text { Saccharification } \\
\%\end{array}$ & $\begin{array}{c}\text { Biomass } \\
(\mathrm{g} / \mathrm{L})\end{array}$ & $\begin{array}{c}\text { Cellulase } \\
\text { activity } \\
(\mathrm{U} / \mathrm{ml})\end{array}$ & rBc & \\
\hline Cotton stalks & $45.87^{\mathrm{b}}$ & $1.39^{\mathrm{b}}$ & $0.94^{\mathrm{b}}$ & 0.89 & 0.93 \\
\hline Corn cobs & $49.68^{\mathrm{a}}$ & $1.66^{\mathrm{a}}$ & $1.02^{\mathrm{a}}$ & 0.88 & 0.92 \\
\hline
\end{tabular}

Therefore, alkali pretreatment has become one of the most promising methods used to degrade lignin in biomass, decrease the polymerization and crystallini structure of cellulose and thus to ease the enzymatic hydrolysis process (Jeya et al., 2009 and Parameswaran et al., 2010). Alkaline pretreatment is the most effective methods compare with acid (Shuhaida and Soh, 2016). The alkali pretreatment can result in a sharp increase in saccharification yields, pretreatment using $\mathrm{NaOH}$ is one of the effective pretreatments, and could digest the hardwood from $14 \%$ to $55 \%$ by reducing the lignin composition from 55\% to 20\% (Balat, 2011 and Behera et al., 2014). Also, Hashem et al. (2013) was proved that lignocellulosic biomass could not be enzymatically saccharified to high yields without a pretreatment, mainly because the lignin in plant cell walls forms a barrier against enzymatic attack.

In addition, Nathalie et al. (2003) discussed the main effect of sodium hydroxide pretreatment on lignocellulosic biomass is delignification by 
breaking the ester bonds cross-linking lignin and xylan, thus increasing the porosity of biomass. Iroba et al. (2013) and Cabrera et al. (2014) also stated that physicochemical pretreated with $\mathrm{NaOH}$ is the most effective method to break down the lignin.

However, Hashem et al. (2013) reported that non-crystalline and microcrystal cellulose dissolved in $8-10 \% \mathrm{NaOH}$ aqueous solution, indicating the increase of the electron cloud density, as a result of the interactions between cellulose chains with $\mathrm{NaOH}$, which led to the rupture of the intermolecular hydrogen bonds. On that basis, cellulose filaments with small swelling ratio and low fibrillation nature in water were spun (Sen et al., 2016).

From the previous results, it could be conducted that pretreatment of lignocelluloses with $\mathrm{NaOH}$ was the best one. Also, corn cobs was better agricultural waste than cotton stalks for saccarification and cellulase production by mixed fungal cultures of $T$. viride $\mathrm{T} 3 \&$ A niger $\mathrm{A} 4$ as compared to another wastes and another fungi.

So, it could be stated that alkaline pretreatment and mixed culture $T$. viride T3 \& A. niger A4 were selected for further studies.

\section{REFERENCES}

Bahaa, T.; Shawky, M.; Mahmoud, E.; Ghazy, M. and Askerb, M. (2011). Enzymatic hydrolysis of rice straw and corn stalks for monosugars production. Journal of Genetic Engineering and Biotechnology, 9:59-63.

Balat, M. (2011). Production of bioethanol from lignocellulosic materials via the biochemical pathway: a review. Energy Conversion and Management, 52(2):858-75. 
Barnett, H. and Hunter, B. (1998). The illustrated genera of imperfect fungi. 4th (Edn). Aps Press, Saint Paul, Minnesesota. 218 p.

Behera, S.; Arora, R.; Nandhagopal, N. and Kumar, S. (2014). Importance of chemical pretreatment for bioconversion of lignocellulosic biomass. Renew Sust. Energ, 36:91-106.

Binod, P.; Sindhu, R.; Singhania, R.; Vikram, S.; Devi, L.; Nagalakshmi, S.; Kurien, N.; Sukumaran, R. and Pandey, A. (2010). Bioethanol production from rice straw: an overview. Bioresour. Technol., 101:4767-4774.

Cabrera, E.; Munoz, M.; Martin, R.; Caro, I.; Curbelo, C. and Diaz, A. (2014). Alkaline and alkaline peroxide pretreatments at mild temperature to enhance enzymatic hydrolysis of rice hulls and straw. Bioresource Technology, 167:1-7.

Delgenés, J.; Penaud, V. and Moletta, R. (2002). Pretreatments for the enhancement of anaerobic digestion of solid wastes Chapter 8. In: Biomethanization of the Organic Fraction of Municipal Solid Wastes. IWA Publishing, 201-228

Diener, S.; Chellappan, M.; Mitchell, T.; Dunn, N.; Ward, M. and Dean, R. (2004). Insight into Trichoderma reesei genome content, organization and evolution revealed through BAC library characterization. Fungal Genet. Biol., 41:245:277.

Difco Manual, (1984). Dehydrate culture media and reagent for Microbiology. $10^{\text {th }}$ Ed. DIFCO. Laboratories, Detroit, U.S.A.

El Saeidy, E. (2004). Technological fundamentals of briquetting cotton stalks as a biofuel. Ph.D. Thesis, Fac. Landwirtschaftlich-Gärtnerischen Humboldt., Berlin Univ., 119 pp.

Fang, H. and Xia, L. (2013). High activity cellulase production by recombinant Trichoderma reesei ZU-02 with the enhanced cellobiohydrolase production. Bioresour. Technol., 144:693-697.

Fouad, A.A.; Abdel-Moein, N.M.; Mohamed, A.S. and Ahmed, S.E. (2010). Biochemical studies on some cotton by products part I- chemical constituents and cellulose extraction of Egyptian cotton stalks. American Science J., (6):12-19.

Goswami, P.; Blackburn, R.; El -Dessouky, H.; Taylor, J. and White, P. (2009). Effect of sodium hydroxide pre-treatment on the optical and structural properties of lyocell, Eur. Polym. J., 16: 481- 489. 
Hashem, M.; Ali, E. and Abdel-Basset, R. (2013). Recycling Rice straw into Biofuel "Ethanol" by Saccharomyces cerevisiae and Pichia guilliermondii .J. Agr. Sci. Tech.,15:709-721.

Herbert, D.; Phipps, P.J. and Strange, R.E. (1971). Chemical analysis of microbial cells In: Norris JR, hbbons DJ (eds) Method microbiology. Academc Press, London

IBM® SPSS ${ }^{\circledR}$ Statistics software (2011). Version 19.0, SPSS Inc., Chicago, Illinois.

Ioannidou, O.; Zabaniotou, A.; Antonakou, E.; Papazisi, K.; Lappas, A. and Athanassiou, C. (2009). Investigating the potential for energy, fuel, materials and chemicals production from corn residues (cobs and stalks) by non-catalytic and catalytic pyrolysis in two reactor configurations. Renewable and Sustainable Energy. 13(4):750762.

Iroba, K.; Tabil, L.; Dumonceaux, T. and Baik, O. (2013). Effect of alkaline pretreatment on chemical composition of lignocellulosic biomass using radio frequency heating. Biosystems Engineering, 116(4):385-98.

Jeya, M.; Zhang, Y.; Kim, W. and Lee, J. (2009). Enhanced Saccharification of Alkali-treated rice Straw by Cellulase from Trametes hirsutaand Statistical Optimization of Hydrolysis Conditions by RSM. Biores. Technol.J., 100:5155-5161.

Kaplan, M.; Hammerman, C.; Vreman, H.J.; Stevenson, D.K., Pediatr, J. and Beutler, E. (2001). Acute hemolysis and severe neonatal hyper bilirubinemia in glucose-6-phosphate dehydrogenase-deficient heterozygotes, J. Appl. Tech. Enviro.,1 139:137-40.

Kitcha, S. and Cheirsilp, B. (2011). Screening of Oleaginous Yeasts and Optimization for Lipid Production Using Crude Glycerol as a Carbon Source. Energy Procedia., 9:274-282.

Kolmer, J.; Spaalding, E. and Roshi, H. (1959). Approved laboratory techniques 5th (Edn). Century Grofs Ins, New York, USA.

Lee, I.; Evans, B.; Lane, R. and Wood, J. (1996). Substrate-enzyme interactions in cellulose systems. Bioresource Technology, 58: $163-169$ 
Liu, C. and Wyman, C. (2005). Partial flow of compressed-hot water through corn stover to enhance hemicellulose sugar recovery and enzymatic digestibility of cellulose, Bioresour. Technol., 96:1978-1985.

Mandels, M.; Parrish, F. and Reese, E. (1962). Sophorose as an inducer of cellulase in Trichoderma viride. J. Bacteriol. 83:400-408.

Mandels, M.; Weber, J.; Hijny, G. and Reese, E. (1969). (Eds.), Cellulases and their applications, American Chemical Society, Washington, DC, pp. 391-414.

McMillan, J.; Himmel, M. and Baker, R. (1994). Overend (Eds.), enzymatic conversion of biomass for fuels production, American Chemical Society, Washington, DC, pp. 292-324.

Milala, M.; Shugaba, A.; Zanna, A. and Appollos, B. (2014). Isolation and partial purification of cellulase from Rhizopus stolonifer. ARPN J. Science and Technology. 4(8):433-438.

Mona A.; Medwick, V.; Byrd, J.; and Jameel, H. (2001). Soda-AQ Pulping of Cotton Stalks. TAPPI Pulping Conference and published in the conference proceedings (CD version), Saint Paul, Minnesesota.

Mukhopadhyay, S. and Fangueiro, R. (2009). Physical modification of natural fibers and thermoplastic films for composites. Thermoplastic Composite Materials. J., 22(2):135-162.

Nathalie, D.; Francois, G. and Bernard, K. (2003). The unmasking of lignin structures in wheat straw by alkali. Phytochemistry, 63(5):617623.

Nigam, P.S.; Gupta, N. and Anthwal, A. (2009). Pretreatment of agroindustrial residues. In: Nigam PS, Pandey A, eds. Biotechnology for agro-industrial residues utilization. 1 ed. Netherlands: Springer- Verlag, pp. 13-33.

Noriko, H.; Tetsuo, K. and Mitsuro, I. (2005). Enzymatically produced nanoordered short elements containing cellulose Ibcrystalline domains. Carbohydrate Polymers, 61:191-197.

Parameswaran, B.; Raveendran, S.; Reeta, S.; Surender, V.; Lalitha, D.; Satya, N.; Noble, K.; Rajeev, K. and Sukumaran, A. (2010). Bioethanol production from rice straw: An overview. Bioresource Technology. J., 101:4767-4774. 
Pointner, M.; Kuttner, P.; Obrlik, T.; Jäger, A. and Kahr, H. (2014). Composition of corncobs as a substrate for fermentation of biofuels. Agronomy Research, 12(2):391-396.

Ramadan, E.M.; El-Sawy, M.; Gamal, Rawia, F. and Abdelhady, Hemmat, (1985). Growth parameters of yeast grown on agricultural residues using shake flask as a batch culture. Ann. Agric. Sci. Ain Shams Univ., 30:25-45.

Rana, V.; Eckard, A.D.; Teller, P. and Ahring, B.K. (2014). On-site enzymes produced from Trichoderma reesei RUT-C30 and Aspergillus saccharolyticus for hydrolysis of wet exploded corn stover and loblolly pine. Bioresour. Technol., 154:282-289.

Sen, W.; Ang, L. and Lina, Z. (2016). Recent advances in regenerated cellulose materials. Progress in Polymer Science, 53:169-206.

Shuhaida, H. and Soh, K.G. (2016). Effect of Sodium Hydroxide Pretreatment on Rice Straw Composition. Indian J. Science and Technology, 9(21):0974-6846.

Singh, A.; Tuteja, S.; Singh, N. and Bishnoi, N.R. (2011). Enhanced saccharification of rice straw and hulls by microwave-alkali pretreatment and lignocellulolytic enzyme production. Bioresour Technol., 102:1773-1782.

Srilekha, K.; Yadav, S.; Naseeruddin, G.; Sai, P.; Lanka, L.; Venkateswar, R. (2011). Bioethanol fermentation of concentrated rice straw hydrolysate using co-culture of Saccharomyces cerevisiae and Pichia stipitis. Bioresource Technology. J., 102:6473-6478.

Taherzadeh, M. and Karimi, K. (2007). Enzyme-based hydrolysis processes for ethanol from lignocellulosic materials: A review, BioResources, 2(4):707-738.

Tarkov, H. and Feist, W.C. (1969). A mechanism for im-proving the digestibility of lignocellulosic ma-terials with dilute alkali and liquid ammonia. In: Gould, Cellulases and their Applications. Adv. Chem. Ser., 95:197-217.

Velayudhan, S.S.; Ashok, K.B.; Somasundaram, S.; Ganesan, G. and Solomon, R.D.J. (2014). Seaweed (Eucheuma cottonii) associated microorganisms, a versatile enzyme source for the lignocellulosic biomass processing. International Biodeterioration \& Biodegradation, 96:144-151. 
Yonghao, L.; Chenguang, L.; Fengwu, B. and Xinqing, Z. (2016). Overproduction of cellulase by Trichoderma reesei RUT C30 through batch-feeding of synthesized low-cost sugar mixture Bioresource Technology, 216:503-510.

Zhu, S.; Wu, Y.; Chen, Q.; Yu, Z.; Wang, C.; Jin, S.; Ding, Y. and Wu, G. (2006). Dissolution of cellulose with ionic liquids and its application: a mini-review Green Chem, 8:325-7.

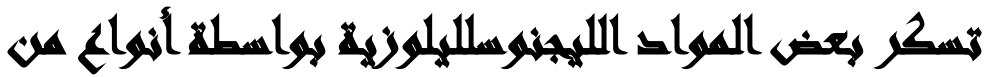

\section{ASPERGILLUS, TRICHODERMA}

[०]

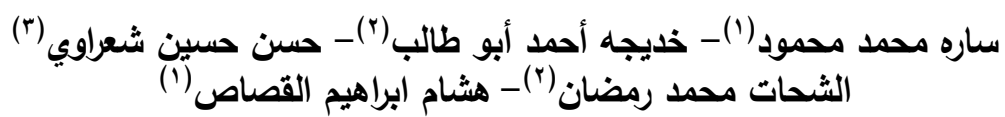

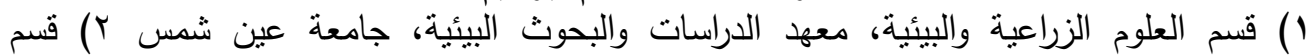

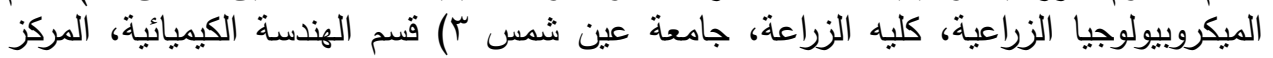
القومي للبحوث

\section{ll}

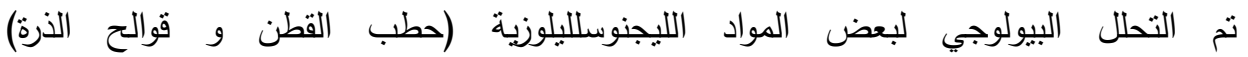
بواسطه (T3) Aspergillus niger وثثلاثة عزلات اخري من (AN2 ، AN2 ، سواء استخدام الفطريات منفردة او مختلطة.

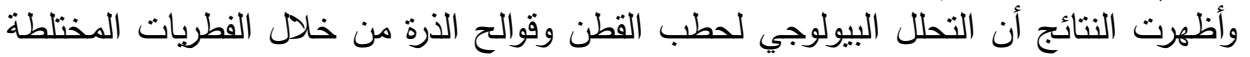

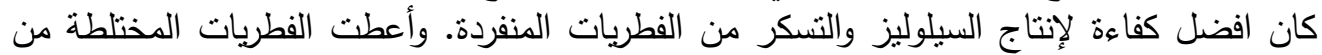
T T. viride T3 \& A. niger A4

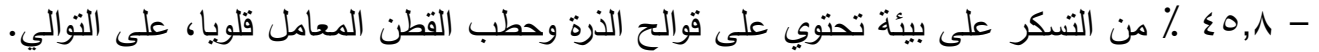

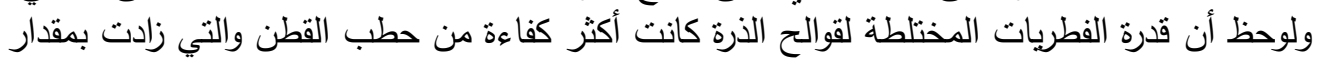

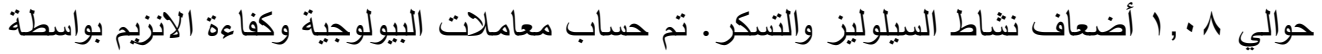

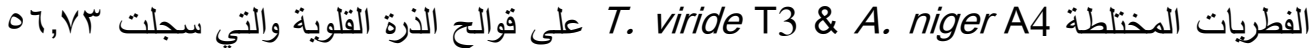

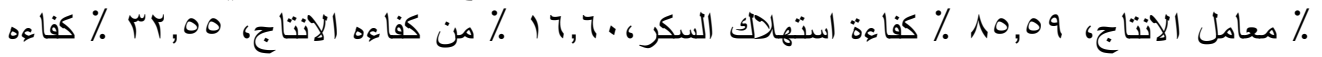

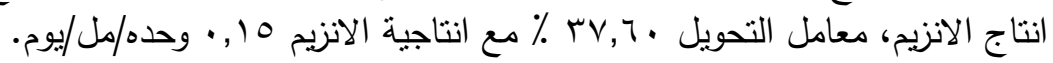
الكلمات الدالة: نشاط السيلوليز، المواد الليجنوسليللوزية، التسكر، Trichoderma viride، .Aspergillus niger 